Draft version SePtember 14, 2018

Preprint typeset using LTEX style emulateapj v. 5/2/11

\title{
DEEP IMAGING OF M51: A NEW VIEW OF THE WHIRLPOOL'S EXTENDED TIDAL DEBRIS
}

\author{
Aaron E. Watkins ${ }^{1}$, J. Christopher Mihos $^{1}$, Paul Harding ${ }^{1}$ \\ Draft version September 14, 2018
}

\begin{abstract}
We present deep, wide-field imaging of the M51 system using CWRU's Burrell Schmidt telescope at KPNO to study the faint tidal features that constrain its interaction history. Our images trace M51's tidal morphology down to a limiting surface brightness of $\mu_{B, \text { lim }} \sim 30 \mathrm{mag} \mathrm{arcsec}^{-2}$, and provide accurate colors $\left(\sigma_{B-V}<0.1\right)$ down to $\mu_{B} \sim 28$. We identify two new tidal streams in the system (the South and Northeast Plumes) with surface brightnesses of $\mu_{B}=29$ and luminosities of $\sim 10^{6} L_{\odot, B}$. While the Northeast Plume may be a faint outer extension of the tidal "crown" north of NGC 5195 (M51b), the South Plume has no analogue in any existing M51 simulation and may represent a distinct tidal stream or disrupted dwarf galaxy. We also trace the extremely diffuse Northwest Plume out to a total extent of 20' (43 kpc) from NGC 5194 (M51a), and show it to be physically distinct from the overlapping bright tidal streams from M51b. The Northwest Plume's morphology and red color $(B-V=0.8)$ instead argue that it originated from tidal stripping of M51a's extreme outer disk. Finally, we confirm the strong segregation of gas and stars in the Southeast Tail, and do not detect any diffuse stellar component in the $\mathrm{H}$ I portion of the tail. Extant simulations of M51 have difficulty matching both the wealth of tidal structure in the system and the lack of stars in the H I tail, motivating new modeling campaigns to study the dynamical evolution of this classic interacting system.
\end{abstract}

Subject headings: galaxies:individual(M51), galaxies:interactions, galaxies:evolution, galaxies:spiral

\section{INTRODUCTION}

Galaxy interactions form the basis of the current hierarchical accretion paradigm for galaxy evolution, and have been linked to the formation of bars, spiral structure, and induced star formation. However, all these features may also result from purely secular processess (see the review by Athanassoula 2010), entangling the various evolutionary effects in individual systems. Understanding interaction histories of nearby galaxies, from which much of our detailed understanding of physical processes comes, is thus particularly important.

The galaxy pair M51 is one of the best known nearby interacting systems, comprised of the grand-design spiral NGC 5194 (M51a) and its early-type SB0 companion NGC 5195 (M51b). Deep imaging in the optical and infrared (e.g. Burkhead 1978, Smith et al. 1990; Mutchler et al. 2005) coupled with kinematic studies in optical (e.g. Tully 1974; Durrell et al. 2003) and radio (e.g. Appleton et al. 1986, Rots et al. 1990), have revealed extended tidal streams and complex kinematics, including an apparently counter-rotating tail of gas on its southern end (Rots et al. 1990).

M51's proximity (7.5 Mpc; Ciardullo et al. 2002, Bose \& Kumar 2014) and well-defined spiral structure has made it a frequent target for studies of the interplay between spiral dynamics, star formation, and the atomic and molecular ISM (e.g. Calzetti et al. 2005; Hughes et al. 2013). If much of the star formation present in the system is interaction-induced, models become an important means of constraining the physical mechanisms and timescales involved, and hence, for example, the calibration of star formation tracers in galaxies (e.g. Calzetti et al. 2005) and determination of generalized star formation laws (e.g. Kennicutt et al. 2007).

This has made the pair a favorite subject of interaction models (e.g. Toomre \& Toomre 1972; Howard \& Byrd 1990; Salo

${ }^{1}$ Department of Astronomy, Case Western Reserve University, Cleveland, OH 44106, USA
\& Laurikainen 2000; Durrell et al. 2003; Dobbs et al. 2010 and many others), constrained by the morphology and kinematics of the observed tidal features. These modeling campaigns have led to two classes of models for the system: one involving a single fly-by passage (Toomre \& Toomre 1972, Hernquist 1990, Durrell et al. 2003), and a second involving multiple passes on a more tightly bound orbit (Salo \& Laurikainen 2000, Dobbs et al. 2010). Discriminating between these scenarios can constrain both the timescale and strength of the perturbation that set up the grand-design spiral and induced star formation in the M51 system.

Deeper observations can prove useful in locating additional interaction signatures to further constrain the models. Particularly in M51, in which both galaxies have vastly different star formation rates (Lee et al. 2011, 2012), broadband colors may be used to help determine the origin of many interaction signatures, and add additional constraints from stellar populations. Combining these observations with the kinematics and spatial distribution of the gas in the system can also greatly narrow down the available parameter space for the dynamical models. In this Letter, we present deep multiband optical imaging of M51, taken as part of a survey of low surface brightness features of nearby galaxies, using the $0.6 / 0.9 \mathrm{~m}$ CWRU Burrell Schmidt Telescope at Kitt Peak National Observatory (Watkins et al. 2014). We report on the morphology and colors of both known and newly-discovered tidal features which will help constrain the dynamical evolution of the M51 system.

\section{OBSERVATIONS AND ANALYSIS}

We give a brief account of our observation and data reduction procedures here; more details can be found in Watkins et al. (2014) and references therein. We observed M51 in spring 2010 and spring 2012, with a setup identical to that described in Watkins et al. (2014). We observed only on moonless photometric nights, in two filters: modified Johnson $B(31 \times 1200$ s exposures $)$ and Washington $M(39 \times 900$ s exposures $)$. We generated dark sky flats using a comparable number of offset 

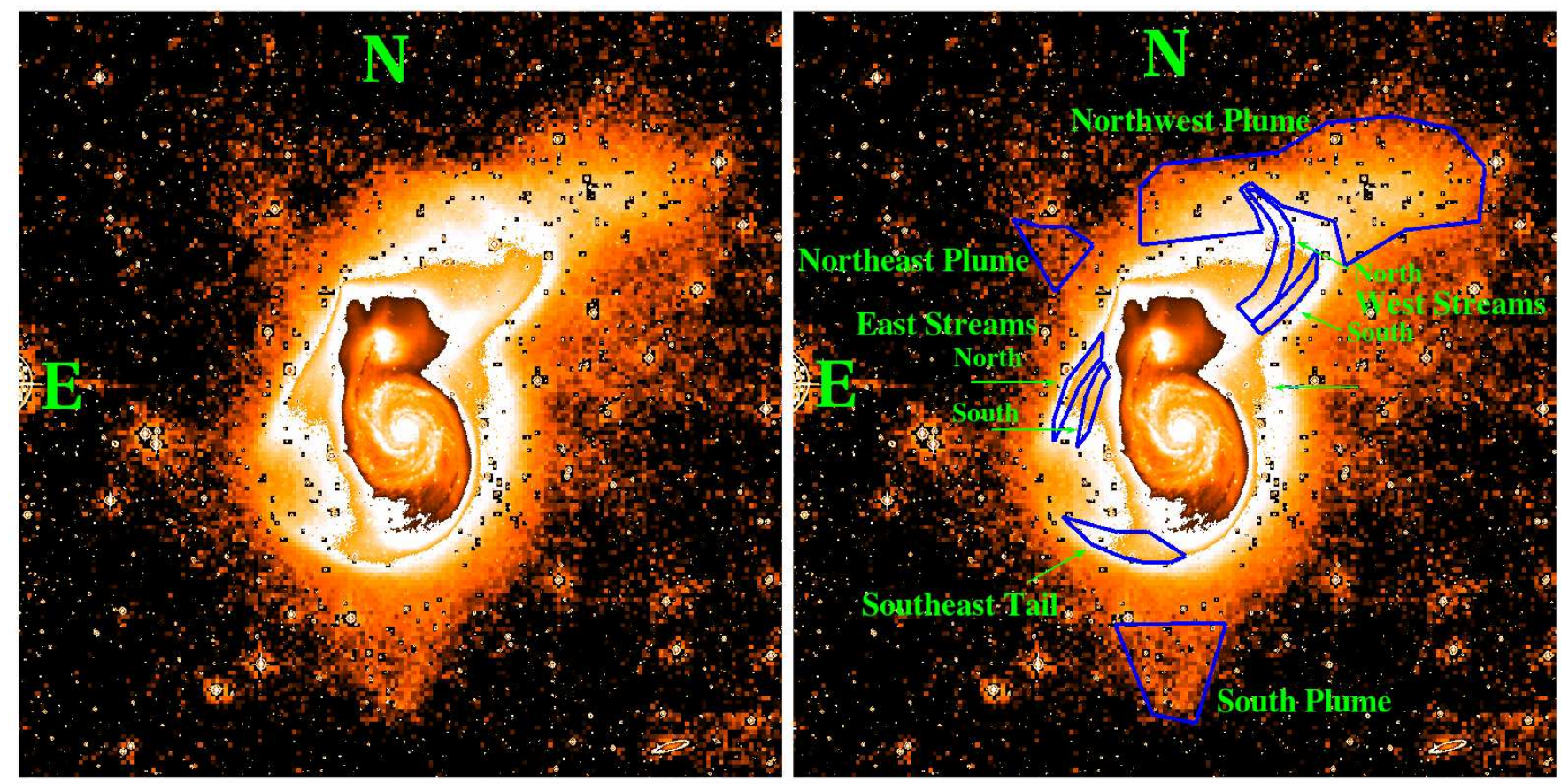

Figure 1. Left: a subset of our B band mosaic, rescaled in intensity for different surface brightness ranges: $\mu_{B}<24.6,24.6<\mu_{B}<26.5$, and $\mu_{B}>26.5$. At the lowest surface brightnesses, the image has been median binned to enhance faint structures. Right: as left, marking regions detailed in Table 1 and discussed in the text. Each image is $39^{\prime}(84 \mathrm{kpc})$ on a side.

Table 1

Photometry of Tidal Debris

\begin{tabular}{lcccccc}
\hline \hline \multicolumn{1}{c}{ Region } & $\mu_{B, p e a k}$ & $M_{B}$ & $B-V$ & $10^{6} L_{\odot, B}$ & Origin & Gas Content \\
\hline West Stream - North & 25.43 & -14.3 & 0.75 & 45 & M51b & Gas Poor \\
West Stream - South & 25.46 & -14.2 & 0.73 & 39 & M51b & Gas Poor \\
East Stream - North & 25.78 & -14.1 & 0.73 & 39 & M51b & Gas Poor \\
East Stream - South & 25.63 & -13.9 & 0.72 & 30 & M51b & Gas Poor \\
Southeast Tail & 25.80 & -14.8 & 0.64 & 68 & M51a & Gas Rich, offset \\
Northwest Plume & 27.59 & -14.5 & 0.81 & 101 & M51a? & Patchy? \\
Northeast Plume & $29.0 \pm 0.3$ & -11.2 & - & 4 & M51a? & Gas Poor \\
South Plume & $29.2 \pm 0.7$ & -11.8 & - & 8 & Unknown & Gas Poor \\
\hline
\end{tabular}

Note. - Absolute photometric errors are $\sigma_{B} \sim 0.04$ and $\sigma_{B-V} \sim 0.05$.

night sky images in each filter (Watkins et al. 2014). Photometric conversion to standard Johnson $B$ and $V$ used observations of Landolt (1992) standard fields to determine color terms, and on-frame SDSS (DR8; Aihara et al.2011) stars to derive direct photometric zeropoints.

Data reduction consisted of standard reduction procedures and modeling and subtraction of reflections and extended wings of bright stars (see Slater et al. 2009). Following sky subtraction, each image was spatially registered and scaled to a common photometric zeropoint before median combining all images into the final $B$ and $V$ mosaics. We then masked the final mosaics of bright discrete sources and spatially rebinned and median filtered them in $9 \times 9$ pixel blocks to enhance faint, diffuse features. Our final $B$ image and our colormap of M51 are shown in Figures 1 and 2 , respectively. Our limiting magnitude is $\mu_{B} \sim 30$.

In the quantitative photometric analysis that follows, we use polygonal apertures to define regions of interest in M51 (see Figure 1 and Table 1 ). In these regions, contamination from background sources can be significant, so we subtract off a local background flux (see Rudick et al. 2010; Mihos et al. 2013, for details). At extremely low surface brightnesses, this background contamination dominates the uncertainty, and is non-Gaussian. At surface brightnesses of $\mu_{B}<27.5$, the relative uncertainties are small, but at extremely low surface brightness $\left(\mu_{B}>28.5\right)$ they become significant and preclude measurement of meaningful colors. All values have also been corrected for the local foreground extinction $\left(A_{B}=0.126\right.$, $E(B-V)=0.031$; Schlafly \& Finkbeiner 2011).

In searching for tidal features in deep surface photometry, an additional source of contamination is scattered light from foreground Galactic dust, which can mimic diffuse structures (see Rudick et al. 2010). However, such dust radiates in the mid- and far-infrared, and a comparison of our images with the WISE $12 \mu \mathrm{m}$ and IRIS $100 \mu \mathrm{m}$ images (Meisner \& Finkbeiner 2014; Miville-Deschênes \& Lagache 2005, respectively) shows no evidence for strong dust contamination cospatial with the diffuse features we identify in Figure 1 (to 


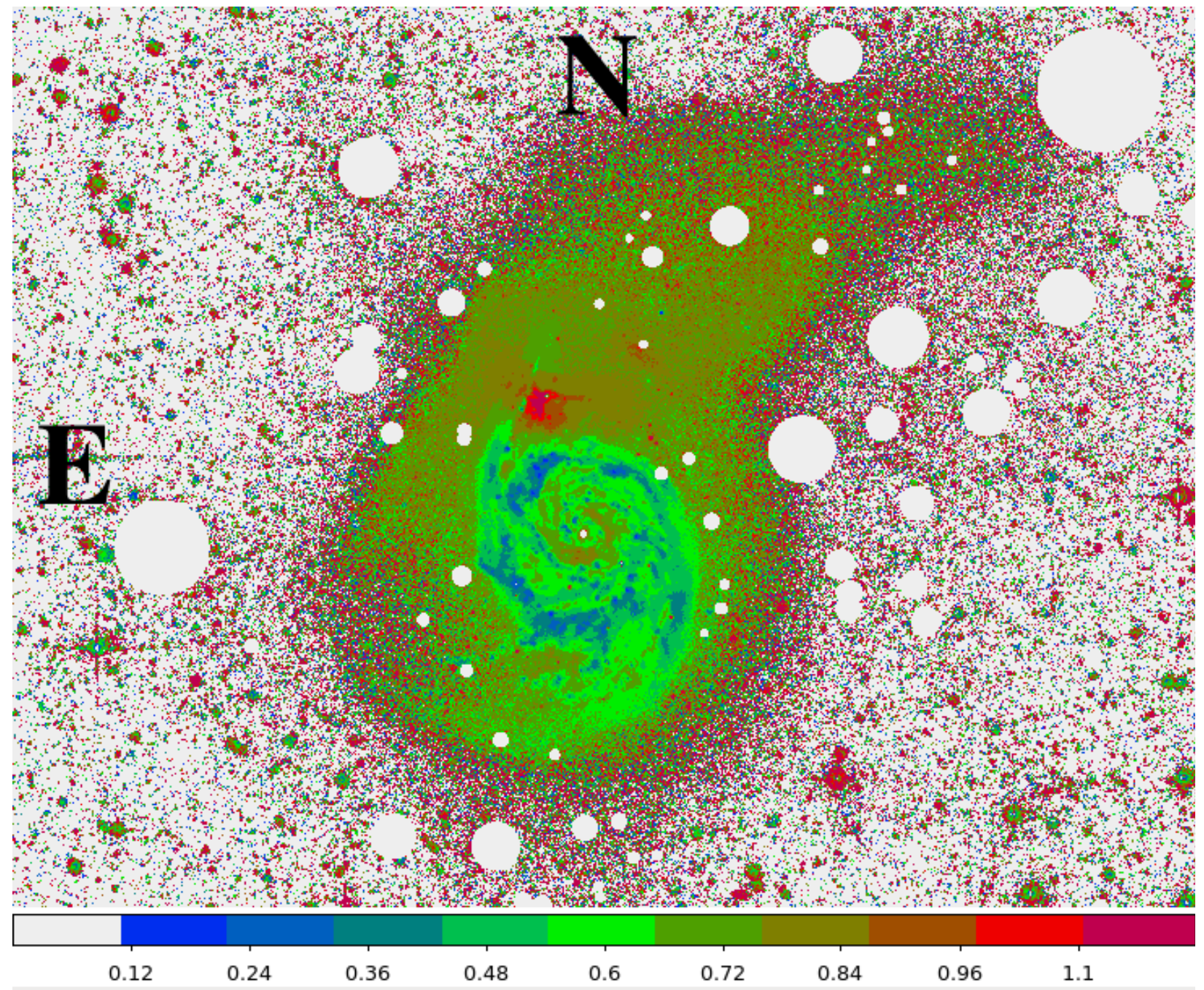

Figure 2. B-V colormap of the M51 system. The image spans $36^{\prime} \times 27^{\prime}(78 \mathrm{kpc} \times 58 \mathrm{kpc})$.

limits of 3.58MJy $s r^{-1} / 1.11 M J y s r^{-1}$ in WISE/IRIS).

\section{RESULTS}

\subsection{Morphology and color of tidal features}

An examination of Figure 1 shows a wealth of tidal debris around M51. At higher surface brightness $\left(\mu_{B}<26.5\right)$ we see many well-known features, including M51's southern tidal tail, the east and west tidal streams emanating from M51b's disk, and the three-pronged structure (the "crown") just north of M51b. At extremely low surface brightness, we trace the extended Northwest Plume, and identify two new, extremely faint features - the Northeast and South Plumes. We also show in Figure 3 a comparison between our optical image and the $\mathrm{H}$ I data from Rots et al. (1990).

The most extended feature is the Northwest Plume. Originally detected by Burkhead (1978), its tip lies 20' (43 kpc) from the center of M51a. While it partially blends with the brighter West Streams extending from M51b, a variety of arguments indicate it is a separate feature. First, the Plume is morphologically distinct-it extends almost directly west, while the northern West Stream extends first northwest and then curves toward the northeast (Figure 11). The Northwest Plume is also $\sim 0.06$ magnitudes redder ${ }^{2}$ than the adjacent

${ }^{2}$ While this difference is comparable to our absolute photometric uncer-
West Streams (Table 1, we note that the plume shows a uniform color from end to end), implying distinct (albeit similar) stellar populations. Durrell et al. (2003) also note bimodality in the PNe velocities northwest of M51 (although not cospatial with the Plume), arguing for kinematically distinct populations. Indeed, we note a possible third component, a slight protrusion just to the northwest of M51a (marked with an arrow in Figure 1) which may be a stellar stream overlapping M51a's outer disk. The THINGS H I map of M51 (Walter et al. 2008) also shows gas extending roughly in this direction.

There is very little high column density $\mathrm{H}$ I associated with the Northwest Plume (Figure 3, but evidence exists for extremely diffuse, high-velocity gas in the vicinity (Appleton et al. 1986; Rots et al. 1990, Pisano private communication). Diffuse H I associated with the Northwest Plume would also imply an origin with the gas-rich spiral M51a, as the companion galaxy M51b appears to be extremely gas-poor.

Dynamically, the Northwest Plume appears several hundred Myrs old; its linear extent ( $\sim 40 \mathrm{kpc}$ in projection) would require an unrealistic expansion velocity if, for example, it had formed during the most recent passage proposed by Salo \& Laurikainen (2000) (50 to $100 \mathrm{Myr}$ ago) or Howard \& Byrd

tainty, relative color differences between adjacent features are constrained to $\sim 0.01$ mag uncertainty, due to the stability of the background corrections. 


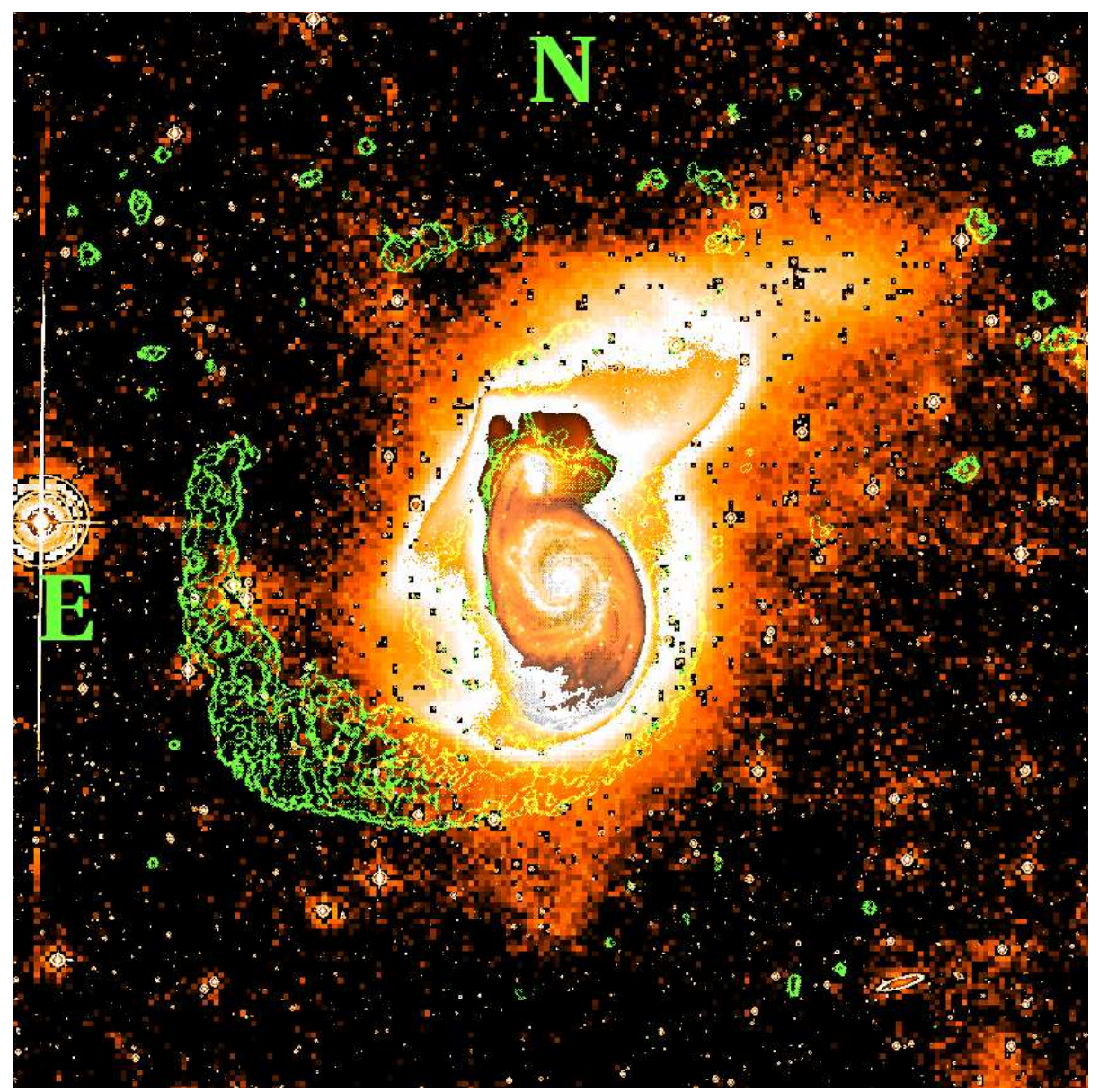

Figure 3. Comparison between our optical image and (in green contours) the $\mathrm{H}$ I data of Rots et al. (1990).

(1990) (70 Myr ago). At the rotation speed of M51a (210 $\mathrm{km} / \mathrm{s}$; Appleton et al. 1986), a timescale of 200 Myr is more likely. We have also used stellar population modeling (Kotulla et al.2009) to show the Plume is dominated by old stars. Exponentially declining star formation histories typical of $\mathrm{Sb} / \mathrm{Sc}$ galaxies $(\tau=5-9$ Gyr) produce colors too blue $(B-V \lesssim 0.6)$ to match the Plume; its red color demands much older star formation histories ( $\tau=3 \mathrm{Gyr}$ ). Similarly, any subsequent interaction-induced star formation in the Plume must be weak ( $<5 \%$ of the Plume's stellar mass), else the colors would too blue by $>0.15$ mags even after $500 \mathrm{Myr}$. As dust extinction appears negligible due to the low gas column density (Appleton et al. 1986, Rots et al. 1990), our inference of old stellar populations appears sound.

Partially overlapping with the Northwest Plume, but with contrasting morphology and color, are the East and West Streams. Both streams are bifurcated, and all branches have very similar colors $(B-V=0.73)$, which are in turn similar to M51b's average color $(B-V=0.7$; Lee et al. 2012). Given this, and the streams' symmetry around and proximity to $\mathrm{M} 51 \mathrm{~b}$, it seems likely that these streams originated from that galaxy's disk. A variety of processes may give rise to bifurcated tidal streams, including kinematic caustics (Struck \& Smith 2012, Smith et al. 2014) and differences in the kinematic evolution of the gas and stellar components (Mihos 2001). In the latter mechanism, stellar bifurcations would only arise via subsequent star formation in the displaced gas tail, which would then show younger stellar populations than the collisionless tail. The lack of H I and the uniformly red colors of these streams would seem to argue against such a scenario in M51b, and instead favor collisionless kinematic caustics.

Based on the colors shown in Figure 2, much of the crown north of M51b also appears associated with the companion, excepting the bluer middle prong. Lee et al. (2012) showed that the latter is an extension of M51a's northern tidal arm; indeed, its color is similar to that of the Southeast Tail $(\mathrm{B}-\mathrm{V}=0.64)$, the blueness of which is expected from its origin in M51a's disk. We also note the incredible sharpness of the ridge formed by the crown's eastern prong, indicative of a strong caustic.

In the Southeast Tail, the clear separation between the gas and stars (Figure 3, noted previously by Howard \& Byrd (1990) and Rots et al. (1990)) is of particular interest. The stellar tail curves sharply northward and disappears into the tidal detritus near the East Streams, while the more loosely wrapped $\mathrm{H}$ I tail is much more extended and contains no diffuse stellar counterpart down to our limit of $\mu_{B}=30.5$. Simulations have shown that such gas/star offsets in tidal tails can arise from differences in the radial extent of the two components in the pre-encounter disk (Mihos 2001), where the stellar component of a tail consists preferentially of material with low specific angular momentum and thus "trails" the high angular momentum gaseous tail. While this may explain the tail's lack of an old stellar population, the lack of either ongoing star formation (Gil de Paz et al. 2007) or a young stellar population is more challenging, as the compressive nature of tidal interactions is known to form massive star clusters and 
tidal dwarf galaxies (Barnes \& Hernquist 1992; Jarrett et al. 2006 ) in many tidal tails. The lack of a young population in M51's tail may indicate a highly inclined encounter, where compressive forces in the tail are greatly reduced. As shown by Rots et al. (1990), an inclined encounter also explains the tail's observed counter-rotation, and is reproduced well by interaction models of Salo \& Laurikainen (2000).

Aside from the structure of the tidal features, our data also shed light on the structure of M51a's outer disk. The colors get redder by $0.2-0.4$ magnitudes outside of the spiral arm region, visible in the colormap of Figure 2 and explicitly in Figure 4, which shows photometric profiles along an elliptical wedge which follows the curvature of the outermost spiral arm westward into the outer disk. We see a dramatic drop in surface brightness between $220^{\prime \prime}$ and $250^{\prime \prime}$, corresponding precisely with a change in the $\mathrm{B}-\mathrm{V}$ color from 0.46 to 0.65 (roughly, the integrated colors of an Sc and Sa galaxy, respectively; Roberts \& Haynes 1994). This region marks the edge of the outermost spiral arm; beyond, the redward gradient continues, ultimately reaching colors similar to those of M51b. The dramatic drop in surface brightness is similar to the arm-interarm contrast measured in the same region in M51a by Schweizer (1976), and seen in the outer regions of other spiral galaxies (Schweizer 1976; Tacconi \& Young 1990).

The red colors, smooth surface brightness profile, and round outer isophotes in this region might be interpreted as marking the transition to a kinematically hot spheroidal component, but we reject this notion for a variety of reasons. M51a lacks a strong bulge $\left((\mathrm{B}: \mathrm{T})_{I}=0.27\right.$; Pompei \& Natali 1997), arguing that the outer component is not likely to be extended bulge light. Furthermore, such a bright halo component is unlikely given the radius $(\sim 10 \mathrm{kpc})$ and surface brightness $\left(\mu_{B} \approx 25\right)$ of the component; by comparison, M31's smooth halo has the much fainter surface brightness of $\mu_{B} \sim 29-30$ at $10 \mathrm{kpc}$ (extrapolating from the power law fits of Ibata et al. 2014). The most reasonable interpretation for this component is that we are simply seeing the outer, redder stellar disk in M51a.

An outer disk origin for this light is also suggested by its similarity in color to the more extended Northwest Plume. The linear structure of the Plume would require a dynamical origin from material with coherent motion and high angular momentum, as would be found in an outer disk. Such disks are also expected to show redder colors due to effects from radial migration (e.g. Roškar et al. 2008), and such colors are frequently observed in outer disks (e.g. Bakos et al. 2008, who found typical outer disk colors of $g-r \sim 0.5-0.6=0.7-0.8$ in $B-V$ ). Hence, it seems likely that the red outer regions of M51a are simply part of its outer disk, and that the Northwest Plume may have been ejected from the outer disk during the interaction.

\subsection{Comparison to simulations}

Simulations of the M51 system fall broadly into two classes: parabolic single-pass models with typical timescales of > 300 Myr (Toomre \& Toomre 1972; Hernquist 1990; Durrell et al. 2003), and more tightly bound, multiple passage models with one encounter several hundred Myr ago, and a second passage $\lesssim 100$ Myr ago (Howard \& Byrd 1990, Salo \& Laurikainen 2000; Dobbs et al. 2010). In general, these models were developed to match the structure and kinematics of the $\mathrm{H} \mathrm{I}$ and the bright tidal debris (the East and West Streams and Southern Tail). The degree to which they successfully reproduce the fainter, more extended debris (the Northwest, Northeast, and South Plumes) is less clear. Here we develop new insights gleaned from a comparison between our deep imaging data and the existing simulations.

Perhaps most useful is a comparison involving the Northwest Plume. Similar features arise in simulations of M51 from the material nearest the companion at pericenter, which receives the most dispersive tidal kick and is ejected as a broad tidal plume (in contrast, material on the opposite side of the disk forms a coherent tidal tail). Tidal features resembling the Northwest Plume appear in a variety of M51 simulations, but single-passage models tend to reproduce the Plume's morphology better than the best-fit multipassage models of Salo \& Laurikainen (2000) or Dobbs et al. (2010): see, for example, Figures 1 and 8 of (Salo \& Laurikainen 2000; Howard \& Byrd 1990), respectively. However, we note that both Salo \& Laurikainen (2000) and Dobbs et al. (2010) elaborate on only one such model; different parameters in a multi-passage encounter may reproduce the feature more effectively. While the Northwest Plume cannot unambiguously differentiate between the model classes, the dynamical age implied by its linear extent argues that the initial passage of this encounter (or the sole passage) must have taken place at least a few hundred Myr ago (in more agreement with Howard \& Byrd 1990).

Unfortunately, the other faint features - the Northeast and South Plumes-have no clear analogues in any published simulation. The Northeast Plume perhaps most resembles the diffuse northern component seen in the multiple passage models by Salo \& Laurikainen (2000). Single passage models generally completely fail to reproduce this northern component (Salo \& Laurikainen 2000, Durrell et al. 2003), so the presence of both the crown and this Northeast Plume seem to favor the multiple-passage models. Indeed, this feature may simply be a faint outer extension of material in the crown itself; its low luminosity $\left(\sim 10^{6} L_{\odot, B}\right)$ would place it below the mass resolution of most published simulations, and could explain why they miss this feature.

The South Plume, on the other hand, shows no morphological continuity with any adjacent feature, either in optical or in $\mathrm{H} \mathrm{I}$, and has no counterpart in any simulation published to date. While Appleton et al. (1986) detected some diffuse H I on the galaxy's southwestern side, it does not overlap with the South Plume itself. Given all this, the Plume would seem to be either a distinct tidal feature not replicated in previous dynamical modeling (although like the Northeast Plume, it may be too low mass to discern in published simulations), or the remains of a disrupted low luminosity dwarf galaxy in the system.

Regarding the Southeast H I tail, the lack of an old, cospatial stellar component may argue that the gas came from an extended gas-rich disk belonging to M51a. Simulations of the M51 system favor a highly inclined encounter to explain the kinematics of the tail (Rots et al. 1990; Salo \& Laurikainen 2000); such a configuration would suppress caustic formation in the tail and explain its paucity of star formation and young stars. However, the gas/star offset observed in the tail is still problematic, as such features should be most prominent in highly prograde encounters where the orbital and rotational angular momentum is well-aligned (Mihos 2001). Thus the data still leave some ambiguity in the dynamical evolution of the Southeast Tail.

In summary, no single model of M51 provides a perfect match to the additional constraints derived from our imaging. The single-passage models appear to better reproduce 


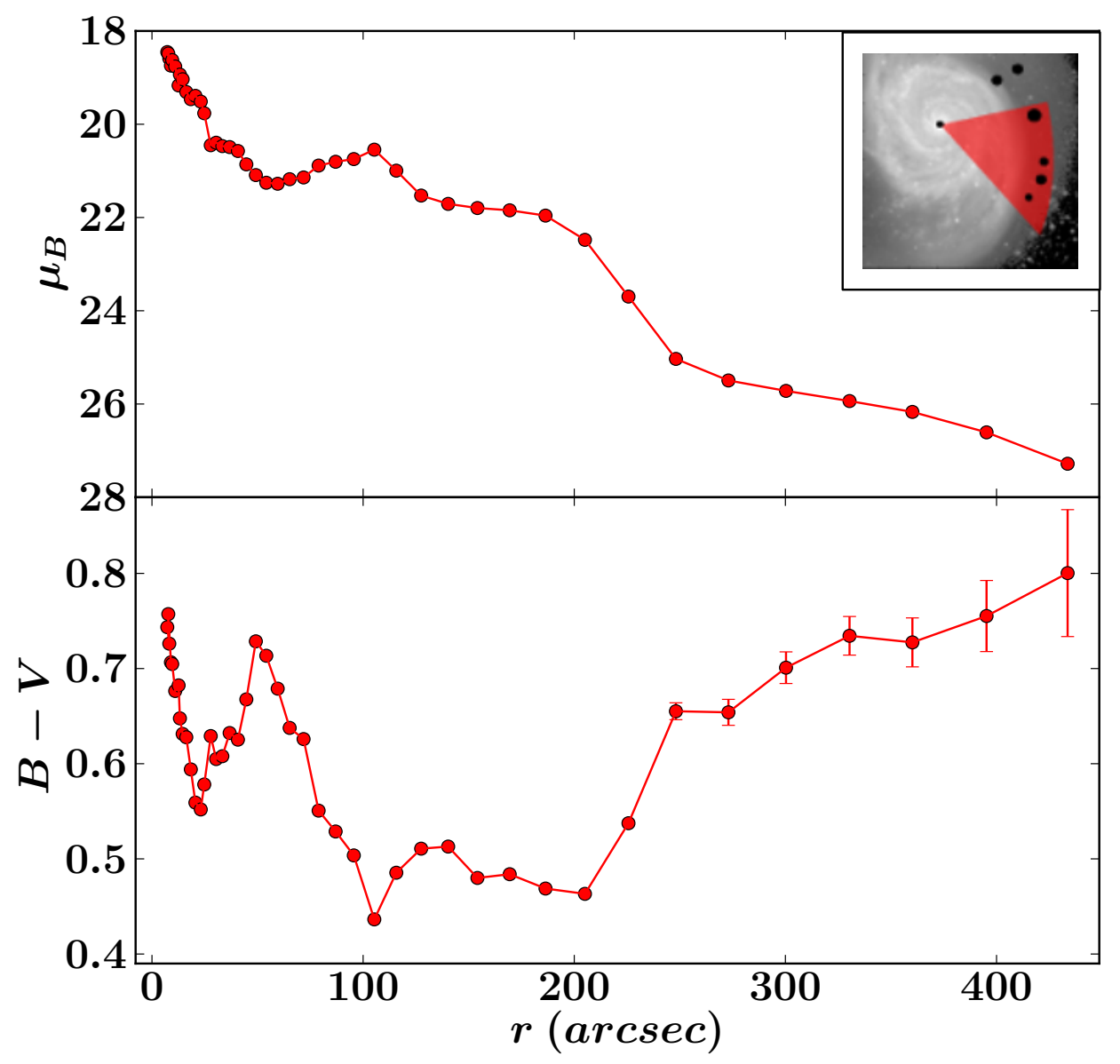

Figure 4. Surface brightness and color profiles along the wedge highlighted in the inset, plotted as a function of the average radial distance from M51a's center. Error bars in both plots are calculated using the background variance, and are often smaller than the point size.

the diffuse Northwest Plume, while the multi-passage models reproduce better the diffuse northern material (the crown and Northeast Plume). No simulations to date have produced anything resembling the South Plume, although its low luminosity suggests that higher resolution simulations may be necessary to model its structure. The lack of a stellar counterpart in M51's Southeast HI tail argues that the tail was drawn almost exclusively from the outermost, gas-rich regions of M51a's disk, or that extensive kinematic segregation of stars and gas has occurred, without triggering any wide-spread star formation in the tidally stripped gas.

Future modeling campaigns thus are warranted, and have a variety of new constraints and challenges ahead. New simulations must recover the newly discovered tidal plumes, ensure that the Northwest Plume is correctly oriented and gasfree, reproduce the strong offset between the stars and gas in the Southeast Tail, and bifurcate M51b's East and West Streams. Successful simulations will then be able to pin down the timescale and geometry of the encounter-critical inputs to studies of spiral structure, ISM evolution, and star formation in this classic interacting system.

The work was supported by the NSF through grant 1108964 to JCM.

\section{REFERENCES}

Aihara, H. et al. 2011, ApJS, 193, 29

Appleton, P. N., Foster, P. A., \& Davies, R. D. 1986, MNRAS, 221, 393

Athanassoula, E. 2010, Galaxies in Isolation: Exploring Nature Versus

Nuture (ASP Conf. Ser. 421), ed. L. Verdes-Montenegro et al. (San

Francisco, CA: ASP), 157

Bakos, J., Trujillo, I., \& Pohlen, M. 2008, ApJ, 683, L103

Barnes, J. E., \& Hernquist, L. 1992, Nature, 360, 715

Bose, S., \& Kumar, B. 2014, ApJ, 782, article id. 98

Burkhead, M. S. 1978, ApJS, 38, 147

Calzetti, D. et al. 2005, ApJ, 633, 871

Ciardullo, R., Feldmeier, J. J., Jacoby, G. H., Kuzio de Naray, R., Laychak, M. B., \& Durrell, P. R. 2002, ApJ, 577, 31

Dobbs, C. L., Theis, C., Pringle, J. E., \& Bate, M. R. 2010, MNRAS, 403, 625

Durrell, P. R., Mihos, J. C., Feldmeier, J. J., Jacoby, G. H., \& Ciardullo, R. 2003, ApJ, 582, 170

Gil de Paz et al. 2007, ApJS, 173, 185

Hernquist, L. 1990, International Conference on Dynamics and Interactions of Galaxies, 108

Howard, S., \& Byrd, G. G. 1990, AJ, 99, 1798

Hughes, A. et al. 2013, ApJ, 779, article id. 46

Ibata et al. 2014, ApJ, 780, 128

Jarrett, T. H. et al. 2006, AJ, 131, 261

Kennicutt, R. C. Jr. et al. 2007, ApJ, 671, 333

Kotulla, R., Fritze, U., Weilbacher, P., \& Anders, P. 2009, MNRAS, 396, 462 Landolt, A. U. 1992, AJ, 104, 340

Lee, J. H., Kim, S. C., Park, H. S., Ree, C. H., Kyeong, J., \& Chung, J. 2011, ApJ, 740, article id. 42 
Lee, J. H., Kim, S. C., Ree, C. H., Kim, M., Jeong, H., Lee, J. C., \& Kyeong, J. 2012, ApJ, 754, 80

Meisner, A. M., \& Finkbeiner, D. P. 2014, ApJ, 781, 5

Mihos, J. C. 2001, ApJ, 550, 94

Mihos, J. C., Harding, P., Spengler, C. E., Rudick, C. S., \& Feldmeier, J. J. 2013, ApJ, 762, article id. 82

Miville-Deschênes, M.-A., \& Lagache, G. 2005, ApJS, 157, 302

Mutchler, M., Beckwith, S. V. W., Bond, H., Christian, C., Frattare, L., Hamilton, F., Hamilton, M., Levay, Z., Noll, K., \& Royle, T. 2005, BAAS, 206, 1307

Pompei, E. \& Natali, G. 1997, A\&AS, 124, 129

Roberts, M. S., \& Haynes, M. P. 1994, ARA\&A, 32, 115

Roškar, R., Debattista, V. P., Stinson, G. S., Quinn, T. R., Kaufmann, T. \& Wadsley, J. 2008, ApJ, 675, L65

Rots, A H., Bosma, A., van der Hulst, J. M., Athanassoula, E., \& Crane, P. C. 1990 , AJ, 100, 387

Rudick, C. S., Mihos, J. C., Harding, P., et al. 2010, ApJ, 720, 569
Salo, H., \& Laurikainen, E. 2000, MNRAS, 319, 377

Schlafly, E. F., \& Finkbeiner, D. P. 2011, ApJ, 737, article id. 103

Schweizer, F. 1976, ApJS, 31, 313

Slater, C. T., Harding, P., \& Mihos, J. C. 2009, PASP, 121, 1267

Smith, J., Gehrz, R. D., Grasdalen, G. L., Hackwell, J. A., Dietz, R. D., \& Friedman, S. D. 1990, ApJ, 362, 455

Smith, B. J., Soria, R., Struck, C., Giroux, M. L, Swartz, D. A., \& Yukita, M. 2014, AJ, 147, 60

Struck, C., \& Smith, B. J. 2012, MNRAS, 422, 2444

Tacconi, L. J., \& Young, J. S. 1990, ApJ, 352, 595

Toomre, A., \& Toomre, J. 1972, A\&A, 178, 623

Tully, R. B. 1974, ApJS, 27, 415

Walter, F., Brinks, E., de Blok, W. J. G., Bigiel, F., Kennicutt, R. C. Jr., Thornley, M. D., \& Leroy, A. 2008, AJ, 136, 2563

Watkins, A. E., Mihos, J. C., Harding, P., \& Feldmeier, J. J. 2014, ApJ, 791, 38 